\title{
P02.23. The efficacy of prolotherapy using dextrose-morrhuate for lateral epicondylosis: a pilot randomized controlled trial
}

\author{
D Rabago*, M Ryan, K Lee, A Chourasia, M Sesto, A Zgierska, D Miller, R Kijowski, J Wilson \\ From International Research Congress on Integrative Medicine and Health 2012 \\ Portland, Oregon, USA. 15-18 May 2012
}

\section{Purpose}

Chronic lateral epicondylosis (CLE) is common, expensive and debilitating. A substantial number of patients are refractory to existing therapy and "watchful waiting." Prolotherapy is a CAM injection therapy for chronic musculoskeletal pain including tendinopathy. We assessed dextrose prolotherapy for CLE in a pilot-level study.

\section{Methods}

The study design was a 2-arm non-blinded randomized controlled trial. Group 1 received dextrose prolotherapy and Group 2 was a waitlist control. Nineteen adults seen with at least 3 months of symptomatic CLE in 22 elbows refractory to prior care received ultrasound-guided injections of 20\% dextrose-morrhuate sodium (Group 1) solution at baseline, 4 , and 8 weeks. Waitlist subjects (Group 2) were followed and discouraged from starting new care. Primary outcome measure was Patient-rated Tennis Elbow Evaluation [PRTEE, (100 points) assessed at baseline, 4, 8 and 16 weeks]. Prolotherapy participants were additionally assessed at 32 weeks. Secondary measures included dynamometer-assessed pain free grip strength and participant satisfaction.

\section{Results}

No baseline differences existed between the groups in gender, duration of elbow pain, prior therapy or baseline PRTEE scores. Prolotherapy participants $(n=10)$ reported improved PRTEE composite scores compared to Waitlist $(\mathrm{n}=12)$ at 4 and 16 weeks $(\mathrm{p}<0.05)$, and improved pain and function PRTEE subscale scores $(\mathrm{p}<0.05)$ at 4 and 16 weeks, respectively. Prolotherapy participants reported improvement in composite PRTEE scores from baseline at 16 and 32 weeks of $17.9 \pm 11.64$ and $24.8 \pm 10.58$ points, a difference of $49.7 \%$ and $70.2 \%$ respectively, far in excess of the 11-point PRTEE-based minimal clinical important difference. Grip strength improved in all groups without between-group difference. Satisfaction with prolotherapy was high; there were no adverse events.

\section{Conclusion}

Prolotherapy using dextrose and morrhuate sodium resulted in safe, significant, sustained improvement of PRTEE-based elbow composite, pain and function scores compared to baseline status and waitlist control subjects. The results of this pilot study suggest the need for a definitive clinical trial.

Published: 12 June 2012

\section{doi:10.1186/1472-6882-12-S1-P79}

Cite this article as: Rabago et al.: P02.23. The efficacy of prolotherapy using dextrose-morrhuate for lateral epicondylosis: a pilot randomized controlled trial. BMC Complementary and Alternative Medicine 201212 (Suppl 1):P79. 